\title{
ELECCIONES DE 12 DE JUNIO DE 1994 AL PARLAMENTO ANDALUZ
}

\author{
JUAN MANUEL GOIG MARTÍNEZ \\ Profesor Asociado del Departamento de \\ Derecho Constitucional
}

Facultad de Derecho. UNED 


\section{SUMARIO}

I. Introducción.-II. Campaña electoral.-III. Resultados electoraLES.-1. Datos generales. -2. Datos por circunscripciones electorales. -3. Resultados por partidos y coaliciones.-IV. CONCLUSIONES. 


\title{
ELECCIONES DE 12 DE JUNIO DE 1994 AL PARLAMENTO ANDALUZ
}

\author{
POR \\ JUAN MANUEL GOIG MARTÍNEZ \\ Profesor Asociado del Departamento \\ de Derecho Constitucional \\ Facultad de Derecho. UNED
}

\section{INTRODUCCIÓN}

Un total de 5.389.660 de andaluces están llamados a las urnas el día 23 de junio para la renovación del Parlamento andaluz.

Los 109 Diputados autonómicos a elegir se reparten en 8 circunscripciones, de las cuales, Sevilla, con un mayor censo elegirá 18; Málaga 16; Cádiz 15; Córdoba y Granada elegirán 13 cada una; 12 Jaen y 11 Diputados representarán a las poblaciones de Huelva y Almería.

De los partidos que participan en la contienda electoral se prevée que sólo 4, PSOE, PP, IU-CA-Los Verdes y PA obtengan representación parlamentaria.

\section{CAMPAÑA ELECTORAL}

Con una antelación de 15 días al 12 de junio se inicia la campaña electoral para la elección de los 109 Diputados que formarán el Parlamento andaluz. 
Estas elecciones, que se hacen coincidir con los comicios al Parlamento europeo, tienen la peculiaridad de convertirse en un auténtico test, tanto para el Gobierno Nacional que ha visto perder un elevado número de votos en las últimas elecciones generales, como para los principales partidos de la oposión, que han aumentado sus espectativas de triunfo.

Con un marcado carácter estatal, puesto que el incipiente nacionalismo andaluz se encuentra sometido a una profunda crisis interna, se celebra esta campaña, caracterizada por cuatro aspectos fundamentales: el enfrentamiento personal entre los principales partidos y coaliciones contendientes; la crisis económica e industrial que afecta a Andalucia; la crisis interna que enfrenta a los distintos sectores del socialismo andaluz, y la corrupción, que aparece como trasfondo en todas las actuaciones de campaña.

Uno de los aspectos más importantes de esta campaña es la posible pérdida de la mayoría absoluta del Gobierno socialista que puede pasar incluso, de acuerdo con algunos sondeos preelectorales, a convertirse en la segunda fuerza política, por debajo del PP.

En los sondeos electorales, la coincidencia se centra en la pérdida de la mayoría absoluta que el PSOE ha mantenido desde 1982.

La encuesta de "Diario 16", en consonancia con los resultados que ofrece para las europeas, es la más favorable para las espectativas del PSOE, ya que le otorga una ventaja de casi 14 puntos. El sondeo de wel Mundo" es el que sitúa a los populares más cerca del PSOE en las andaluzas, aunque sacándoles 7,2 puntos.

"El País" otorga a los socialistas 10 puntos de ventaja en el conjunto de las ocho provincias andaluzas, $y$ "La Vanguardia», 6 puntos.

\section{Evolución de la Composición del Parlamento *}

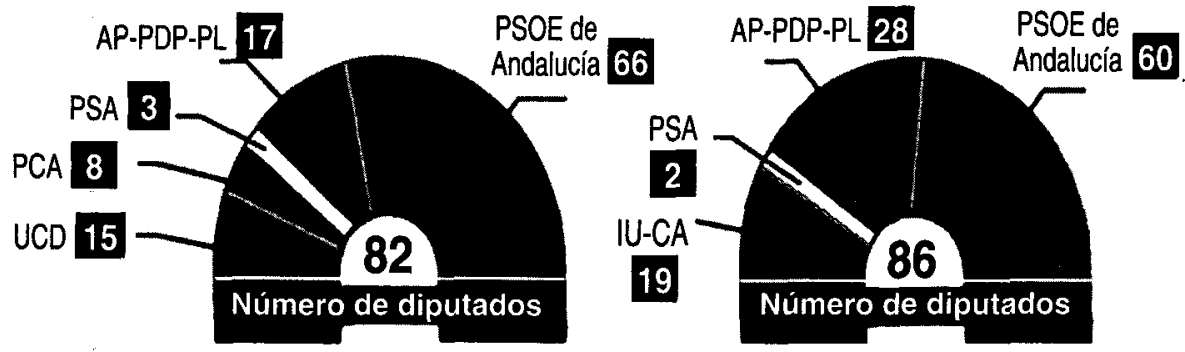

* Gráfico realizado por Olive Estudio para «El Semanal». 12 de junio de 1994. 


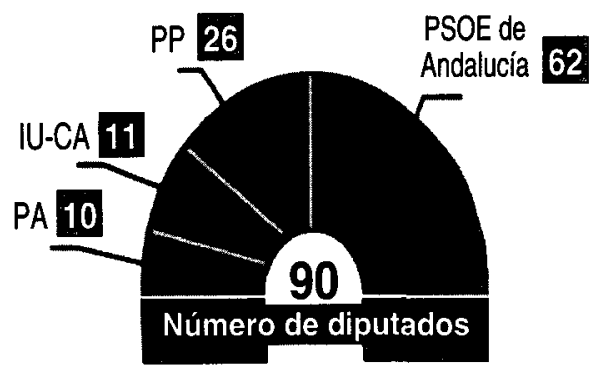

Sondeos Electorales

"El Pais». 5 de junio de 1994. Demoscopia.

\begin{tabular}{ccc}
\hline Partido & $\%$ & Escaños \\
\hline PSOE & 40,7 & $48-51$ \\
\hline PP & 30,9 & $34-37$ \\
\hline IU-CA-V & 18,2 & $18-20$ \\
\hline PA & 6,2 & $3-4$ \\
\hline
\end{tabular}

"Diario 16». 5 de junio de 1994. ICP-Research.

\begin{tabular}{ccc}
\hline Partido & $\%$ & Escaños \\
\hline PSOE & 40,6 & $46-48$ \\
\hline PP & 26,8 & $30-33$ \\
\hline IU-CA-V & 19,2 & $17-21$ \\
\hline PA & 5,4 & $3-4$ \\
\hline
\end{tabular}

"El Mundo». 5 de junio de 1994. Sigma Dos.

\begin{tabular}{ccc}
\hline Partido & $\%$ & Escaños \\
\hline PSOE & 39,4 & $45-49$ \\
\hline PP & 32,2 & $38-40$ \\
\hline IU-CA-V & 17,8 & $17-20$ \\
\hline PA & 6,9 & $5-3$ \\
\hline
\end{tabular}


"La Vanguardia». 5 de junio de 1994. Instituto Opina.

\begin{tabular}{ccc}
\hline Partido & $\%$ & Escaños \\
\hline PSOE & 40,0 & $45-49$ \\
\hline PP & 34,0 & $37-41$ \\
\hline IU-CA-V & 15,0 & $14-16$ \\
\hline PA & 11,0 & $5-4$ \\
\hline
\end{tabular}

Las pretensiones de las principales coaliciones y partidos que intervienen en los comisios puede sintetizarse de la siguiente forma:

PSOE. El temor a la pérdida de la mayoría absoluta y a un posible voto de castigo desde la izquierda, pronosticado por el crecimiento que los sondeos electorales atribuyen a IU, lleva al Partido del Gobierno andaluz a la búsqueda del voto útil, bajo el argumento de representar a la «izquierda real», buscando "el uso de la razón» ?.

EI PSOE defiende su gestión al frente de la Junta de Andalucía, ponderando los logros en infraestructuras, carreteras, educación y sanidad, e intenta evitar una fuerte derrota, consecuencia de los problemas económicos, la corrupción y la división interna del partido.

El programa electoral del PSOE, cuya medida más polémica ha sido, sin duda, la promesa de vacaciones pagadas para las amas de casa andaluzas, persigue el pleno desarrollo del Estatuto de Andalucía y la promoción de esta Comunidad en Europa, si bien en algunos momentos este programa ha quedado en un segundo plano en los actos electorales, en favor de los ataques $y$ descalificaciones a los partidos opositores ${ }^{2}$.

PP. De "promesas fáciles, demagógicas y electoralistas" ${ }^{3}$ califica el PP el programa electoral del PSOE, al que acusan de incumplimiento de programas anteriores $y$ de intentar comprar el voto andaluz a través del PER (Plan de Empleo Rural).

\footnotetext{
"El País» 6 de junio de 1994.

2 "ABC" 10 de junio de 1994.

3 "ABC" 9 de junio de 1994.
} 
La enorme escalada que los sondeos electorales vaticinan para el Partido Popular llevan al principal partido opositor a intentar conseguir un efecto de arrastre para ampliar al máximo el margen de la victoria ${ }^{4}$.

La pacificación de las instituciones, la recuperación de la debilitada economía andaluza y la consecución de un mayor poder para Andalucía en el Estado español y en la Unión Europea, son los puntos fundamentales del programa electoral del PP.

El enfrentamiento personal entre los representantes de los principales partidos mayoritarios culmina con la presentación de una querella por injurias contra Alfonso Guerra, por las acusaciones que hace a Javier Arenas de ocultamiento de actividades empresariales.

\section{IU - Convocatoria por Andalucía - Los Verdes}

La coalición que lidera en Andalucía Luis Carlos Rejón aprovecha el avance que pronostican los sondeos al PP para pedir más apoyos para IU.

La lucha de IU en estos comicios viene centrada en la consecución de unos resultados suficientes para acabar con el bipartidismo y que le permitan actuar de llave para la gobernabilidad de la Comunidad andaluza para lo que ofrecen un programa que persigue la recuperación económica, el desarrollo social en Andalucía y recobrar el prestigio de la vida política.

Desde las listas de esta coalición, $y$ ante los pronósticos electorales, se niega un posible pacto a la griega (IU - PP), así como un pacto PSOE IU para el supuesto de que el partido gobernante perdiera la mayoría absoluta, al que acusan de ser el causante de los problemas económicos que soporta Andalucia, de debilidad y de utener una comunidad de valores con el PP» ${ }^{5}$.

$P A$. La posible concentración del voto, el voto útil y los augurios de las encuestas electorales son el principal escollo con que se encuentra el PA, que podría ver reducidos sus 10 escaños a 3 ó 4 .

Las acusaciones de ineficacia y centralismo hacia el PSOE son los aspectos principales de su campaña, en la que, por encima de todo, in-

5 Carlos Pastor. "El País" 6 de junio de 1994. 
JUAN MANUEL GOIG MARTÍNEZ

tenta dar solución a los problemas económicos y a las corrupte las surgidas en Andalucía.

En andalucismo, que ha experimentado una vida "guadanesca" 6 lucha por mantener su actual posición de privilegio político en el Parlamento andaluz; busca adoptar un planteamiento de consenso, $y$ apuesta por la gran unidad de los nacionalismos andaluces?.

III. RESULTADOS ELECTORALES

Sin apenas incidentes se desarrolló la jornada electoral, jornada que arrojó unos resultados esperados, a la vista de los sondeos electorales, de los cuales quizás, el más importante venga determinado por el índice de participación.

Participación*

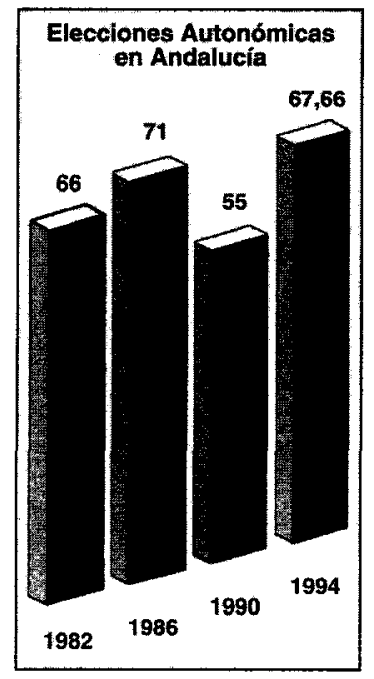

- Lourdes Lucio. "El País» 13 de junio de 1994.

7 "El Mundo". Declaraciones de Rojas Marcos. 13 de junio de 1994.

* Extraído de «El País». 14 de junio de 1994.

276 
Aunque algo inferior al porcentaje obtenido en las consultas autonómicas de 1986, el 67,66 por 100 de participación supera en más de 12 puntos la experimentada en 1990, lo que puede ser entendido como un deseo del electorado andaluz por modificar la situación vivida por Andalucía durante los últimos cuatro años, época en la que la falta de interés por los asuntos políticos propició una de las abstenciones más fuertes en el panorama electoral español.

La intención de castigo hacia el partido gobernante ha llevado al ciudadano andaluz a manifestar su descontento y a la búsqueda de nuevas espectativas de gobierno.

\section{Datos generales}

Censo:

5.389 .660

Votos nulos: $\quad 21.757(0,60 \%)$

Votos en blanco: $30.709(0,85 \%)$

Escaños:

109

Participación: $\quad 67,66 \%$

\begin{tabular}{cccccccc}
\hline & & \multicolumn{1}{c}{1994} & & \multicolumn{2}{c}{1990} & \multicolumn{2}{c}{$1990-1994$} \\
\hline Partidos & Escaños & $\%$ & Votos & Escaños & $\%$ & Escaños & $\%$ \\
\hline PSOE & 45 & 38,56 & 1.382 .991 & 62 & 49,61 & -17 & $-11,05$ \\
\hline PP & 41 & 34,47 & 1.236 .005 & 26 & 22,17 & +15 & $+12,30$ \\
\hline IU-CA-V & 20 & 19,17 & 687.415 & 11 & 12,67 & +9 & $+6,5$ \\
\hline PA* & 3 & 5,80 & 208.141 & 10 & 10,75 & -7 & $-4,95$ \\
\hline Otros & - & 2 & 40.954 & - & 4,80 & - & $-2,80$ \\
\hline
\end{tabular}

* Antes Partido Andalucista. 


\section{Datos por circunscripciones electorales}

Circunscripción de ALMERÍA

Censo:

358.849

Participación: $68,60 \%$

Escaños:

11

\begin{tabular}{ccccccccc}
\hline & & \multicolumn{1}{c}{1994} & \multicolumn{2}{c}{1990} & \multicolumn{2}{c}{$1990-1994$} \\
\hline Partidos & Escaños & $\%$ & Votos & Escaños & $\%$ & Escaños & $\%$ \\
\hline PSOE & 5 & 37,82 & 90.827 & 7 & 49,97 & -2 & $-12,15$ \\
\hline PP & 5 & 42,20 & 101.366 & 3 & 28,47 & +2 & $+13,73$ \\
\hline IU-CA-V & 1 & 15,08 & 36.231 & 1 & 9,04 & $=$ & $+6,04$ \\
\hline PA $^{*}$ & - & 3,21 & 7.722 & - & 6,57 & - & $-3,36$ \\
\hline
\end{tabular}

Circunscripción de CÁDIZ

Censo: $\quad 815.676$

Participación: $\quad 60,51 \%$

Escaños: $\quad 15$

\begin{tabular}{cccccccc}
\hline & \multicolumn{3}{c}{1994} & \multicolumn{2}{c}{1990} & \multicolumn{2}{c}{$1990-1994$} \\
\hline Partidos & Escaños & $\%$ & Votos & Escaños & $\%$ & Escaños & $\%$ \\
\hline PSOE & 5 & 34,38 & 166.644 & 8 & 46,60 & -3 & $-12,22$ \\
\hline PP & 5 & 33,33 & 161.528 & 2 & 15,54 & +3 & $+17,79$ \\
\hline IU-CA-V & 3 & 18,25 & 88.444 & 1 & 10,05 & +2 & $+8,20$ \\
\hline PA $^{*}$ & 2 & 11,67 & 56.553 & 4 & 21,58 & -2 & $-9,91$ \\
\hline
\end{tabular}

* Antes Partido Andalucista. 
Circunscripción de CÓRDOBA

Censo:

588.008

Participación:

$73,30 \%$

Escaños:

13

\begin{tabular}{cccccccc}
\hline & \multicolumn{3}{c}{1994} & \multicolumn{2}{c}{1990} & \multicolumn{2}{c}{$1990-1994$} \\
\hline Partidos & Escaños & $\%$ & Votos & Escaños & $\%$ & Escaños & $\%$ \\
\hline PSOE & 6 & 37,33 & 158.357 & 7 & 47,26 & -1 & $-9,93$ \\
\hline PP & 4 & 30,91 & 131.121 & 3 & 20,74 & +1 & $+10,17$ \\
\hline IU-CA-V & 3 & 24,64 & 104.546 & 2 & 18,81 & +1 & $+5,83$ \\
\hline PA $^{*}$ & - & 5,25 & 22.289 & 1 & 9,25 & -1 & -4 \\
\hline
\end{tabular}

Circunscripción de GRANADA

Censo:

630.540

Participación:

$69,67 \%$

Escaños:

13

\begin{tabular}{cccccccc}
\hline & \multicolumn{1}{c}{1994} & \multicolumn{2}{c}{1990} & \multicolumn{2}{c}{$1990-1994$} \\
\hline Partidos & Escaños & $\%$ & Votos & Escaños & $\%$ & Escaños & $\%$ \\
\hline PSOE & 5 & 38,10 & 163.868 & 7 & 48,90 & -2 & $-10,80$ \\
\hline PP & 6 & 38,83 & 166.999 & 4 & 27,91 & +2 & $+10,92$ \\
\hline IU-CA-V & 2 & 16,87 & 72.547 & 1 & 11,76 & +1 & $+5,11$ \\
\hline PA $^{*}$ & - & 4,44 & 19,077 & 1 & 6,17 & -1 & $-1,73$ \\
\hline
\end{tabular}

* Antes Partido Andalucista. 
Circunscripción de HUELVA

Censo:

344.066

Participación:

$62,54 \%$

Escaños:

11

\begin{tabular}{cccccccc}
\hline & \multicolumn{3}{c}{1994} & \multicolumn{2}{c}{1990} & \multicolumn{2}{c}{$1990-1994$} \\
\hline Partidos & Escaños & $\%$ & Votos & Escaños & $\%$ & Escaños & $\%$ \\
\hline PSOE & 5 & 43,99 & 93.470 & 7 & 55,80 & -2 & $-11,81$ \\
\hline PP & 4 & 33,37 & 70.911 & 2 & 22,19 & +2 & $+11,18$ \\
\hline IU-CA-V & 2 & 15,72 & 33.472 & 1 & 9,31 & +1 & $+6,41$ \\
\hline PA $^{*}$ & - & 5,22 & 11.092 & 1 & 8,22 & -1 & -3 \\
\hline
\end{tabular}

Circunscripción de JAEN

Censo:

498.249

Participación:

$75,55 \%$

Escaños:

\begin{tabular}{cccccccc}
\hline & \multicolumn{3}{c}{1994} & \multicolumn{2}{c}{1990} & \multicolumn{2}{c}{$1990-1994$} \\
\hline Partidos & Escaños & $\%$ & Votos & Escaños & $\%$ & Escaños & $\%$ \\
\hline PSOE & 5 & 42,54 & 158.095 & 7 & 51,80 & -2 & $-9,26$ \\
\hline PP & 5 & 35,94 & 133.577 & 4 & 27,17 & +1 & $+8,77$ \\
\hline IU-CA-V & 2 & 15,65 & 58.156 & 1 & 11,47 & +1 & $+4,18$ \\
\hline PA* $^{*}$ & - & 4,11 & 15.294 & - & 5,57 & - & $-1,46$ \\
\hline
\end{tabular}

* Antes Partido Andalucista. 
Circunscripción de MÁLAGA

Censo:

889.249

Participación:

$64,62 \%$

Escaños:

\begin{tabular}{cccccccc}
\hline & & \multicolumn{1}{c}{1994} & \multicolumn{2}{c}{1990} & \multicolumn{2}{c}{$1990-1994$} \\
\hline Partidos & Escaños & $\%$ & Votos & Escaños & $\%$ & Escaños & $\%$ \\
\hline PSOE & 6 & 34,12 & 192.439 & 9 & 49,26 & -3 & $-15,14$ \\
\hline PP & 6 & 36,67 & 206.862 & 4 & 21,98 & +2 & $+14,69$ \\
\hline IU-CA-V & 4 & 22,43 & 126.537 & 2 & 14,57 & +2 & $+7,86$ \\
\hline PA* $^{*}$ & - & 4,67 & 26.328 & 1 & 8,79 & -1 & $-4,12$ \\
\hline
\end{tabular}

Circunscripción de SEVILLA

$\begin{array}{lr}\text { Censo: } & 1.264 .280 \\ \text { Participación: } & 68,81 \% \\ \text { Escaños: } & 18\end{array}$

\begin{tabular}{cccccccc}
\hline & & \multicolumn{1}{c}{1994} & \multicolumn{2}{c}{1990} & \multicolumn{2}{c}{$1990-1994$} \\
\hline Partidos & Escaños & $\%$ & Votos & Escaños & $\%$ & Escaños & $\%$ \\
\hline PSOE & 8 & 41,84 & 359.291 & 10 & 50,37 & -2 & $-8,53$ \\
\hline PP & 6 & 30,70 & 263.641 & 4 & 19,51 & +2 & $+11,19$ \\
\hline IU-CA-V & 3 & 19,50 & 167.482 & 2 & 12,55 & +1 & $+6,95$ \\
\hline PA $^{*}$ & 1 & 5,80 & 49.786 & 2 & 13,59 & -1 & $-7,79$ \\
\hline
\end{tabular}

* Antes Partido Andalucista. 


\section{Resultados por partidos y coaliciones}

PSOE. EI Partido Socialista, que durante los últimos 12 años ha gobernado con una holgadísima mayoría absoluta ha perdido dicha mayoría en el Parlamento Andaluz, lo que le obliga a buscar apoyos para gobernar.

Varias son las causas que pueden observarse en este debil resultado: 1. La tasa de paro en Andalucía ha alcanzado la cota más alta en el primer trimestre de este año ${ }^{8} ; 2$. La industria andaluza no ha logrado contener la caída en picado de su índice de producción ${ }^{9} ; 3$. La crisis interna del PSOE se ve acrecentada en Andalucía, gran feudo del sector "guerrista"; 4. El desgaste político que han propiciado los casos de corrupción que azotan al país.

"La pérdida de la mayoría absoluta en Andalucía significa..., que los votos cautivos han obtenido la carta de libertad $y$ que ha habido voto útil del PSOE para IU..." ${ }^{10}$.

PP. EI Partido Popular ha aumentado sus votos espectacularmente, tal y como indicaban los sondeos electorales. En términos absolutos, el partido que lidera Javier Arenas ha conseguido el mayor incremento en número de escaños, pasando de los 26 que obtuvo en 1990 a los 40 actuales, desbancando al PSOE en alguno de sus feudos tradicionales como Almería y Málaga y ha logrado empatar en número de escaños en otros dos: Cádiz y Jaen.

EI PP ha conseguido no solamente agrupar todo el voto de la derecha y de centro, así como el andalucista, sino también "ha logrado robar los votos de los electores ideológicamente más moderados que apoyaban al partido socialista" " $"$, imponiendo un cambio en la gobernabilidad de Andalucía ${ }^{12}$.

El resultado electoral es el mejor en la historia del centro político de Andalucía, con el que se pone fin a la hegemonía socialista y "con el que

8 La última encuesta de población activa (EPA) cifra el desempleo en más de 885.000 personas $(34,3$ por 100$)$.

9 Los vapuleos de Santana Motors, Gillete. Puleva, precedidos por las secuelas en Andalucia del fracaso de las inversiones de KIO (Fesa-Enfersa) han explotado muy cerca de las elecciones.

10 Antonio Burgos. "Se estropeó la lavadora andaluza" "El Mundo» 13 de junio de 1994.

1 "El País" 13 de junio de 1994.

12 Raul Heras. "El PP impone el cambio". "El Mundo" 13 de junio de 1994. 
se abre una nueva etapa, según declaraciones de su líder, predominada por el diálogo, la tolerancia y el acuerdo" ${ }^{13}$.

\section{IU - Convocatoria por Andalucía - Los Verdes}

La fuerza política que mayor incremento porcentual ha experimentado en las presentes elecciones ha sido esta coalición que ve duplicado su número de escaños.

IU-CA-Los Verdes se ha convertido, con su crecimiento espectacular, en la fuerza política que tiene en sus manos la gobernabilidad de Andalucía, pues sólo su apoyo, expreso o tácito, al futuro ejecutivo permitirá gobernar con tranquilidad los próximos 4 años.

La coalición se punta "un nuevo tanto en el particular libro de récords de las elecciones andaluzas" ${ }^{14}$, superando el techo marcado por Julio Anguita en 1986, que obtuvo un total de 19 escaños.

La victoria de IU se mide por los objetivos que han conseguido: ser la fuerza determinante del Parlamento andaluz, romper la tenaza bipartidista y referenciarse como alternativa, como la fuerza de la izquierda andaluza.

PA. Además del PSOE, la gran derrota en estos comicios autonómicos fue la coalición andalucista Poder Andaluz, que ha sufrido un fuerte descalabro electoral, derrumbándose en toda Andalucía salvo en las provincias de Sevilla y Cádiz, pasando de 10 a 3 Diputados, y ni siquiera el candidato del enésimo proyecto nacionalista, Arturo Moya, ha conseguido escaño.

El hecho de que el PA no haya sido capaz de trasmitir la importancia de un verdadero poder andaluz, y que el andalucismo no haya superado aún la división sufrida antes de las pasadas elecciones generales, pueden ser las causas de esta declave electoral.

\section{CONCLUSIONES}

Ni las previsiones más optimistas del PP, ni las más pesimistas del PSOE podían preveer tal vuelco en el electorado andaluz.

13 Entrevista de Javier Arenas concedida a "El Mundo" 13 de junio de 1994.

14 Lourdes Luclo. "El Pais» 13 de junio de 1994. 
Estos resultados suponen una modificación espectaclar en la correlación de fuerzas. En la caída de los socialistas han influído decisivamente tanto la crisis industrial que azota la comunidad y la elevada tasa de paro, como las cruentas luchas internas entre "renovadores" y "guerristas". La subida de Aznar en Andalucía se expica, a parte de su programa y de su «invisible» intento por solucionar los problemas de Andalucía, también por "el voto trallero contra Felipe González" 15 .

Las nuevas circunstancias políticas andaluzas permiten un vuelco institucional sin precedentes en la historia reciente del Parlamento andaluz, que tendrá los dos primeros Diputados verdes, presentados en coalición con IU.

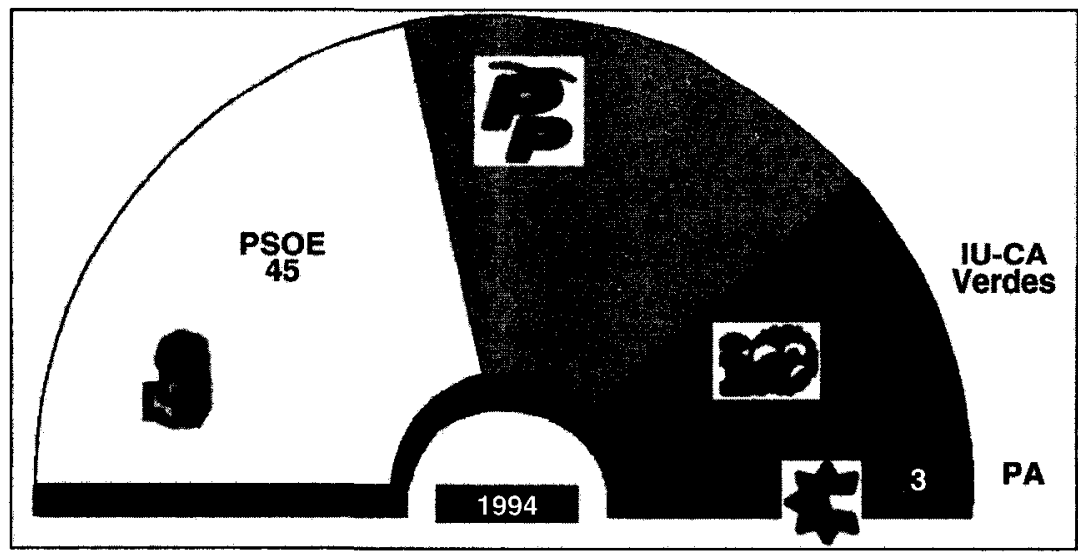

La pérdida de la mayoría absoluta por el PSOE, y el crecimiento del PP por su derecha, y de IU por su izquierda, permitirá que el Pleno de la Asamblea autonómica tenga en sus manos gran parte de los instrumentos necesarios para propiciar una rehabilitación del funcionamiento institucional.

Aunque el número de escaños obtenidos permitirá al Partido Socialista gobernar en minoria, ya que no es previsible un pacto de legislatura IU-PP, será necesario que busque apoyos para temas puntuales con los partidos de la oposición si quiere sacar adelante su programa de gobierno.

15 Francisco Umbral. «El vuelco" "El Mundo" 13 de junio de 1994. 